\title{
Phenomenological Models of the Quark-Gluon Plasma Equation of State
}

\author{
Peter N. Meisinger ${ }^{a *}$, Travis R. Miller ${ }^{\text {a }}$, Michael C. Ogilvie ${ }^{a}$ \\ a Dept. of Physics, Washington University, \\ St. Louis, MO 63130 USA
}

Two phenomenological models describing an $S U(N)$ quark-gluon plasma are presented. The first is obtained from high temperature expansions of the free energy of a massive gluon, while the second is derived by demanding color neutrality over a certain length scale. Each model has a single free parameter, exhibits behavior similar to lattice simulations over the range $T_{d}-5 T_{d}$, and has the correct blackbody behavior for large temperatures. The $N=2$ deconfinement transition is second order in both models, while $N=3,4$, and 5 are first order. Both models appear to have a smooth large- $N$ limit. In both models, the confined phase is characterized by a mutual repulsion of Polyakov loop eigenvalues that makes the Polyakov loop expectation value zero. In the deconfined phase, the rotation of the eigenvalues in the complex plane towards 1 is responsible for the approach to the blackbody limit over the range $T_{d}-5 T_{d}$. The addition of quarks in $S U(3)$ breaks $Z(3)$ symmetry weakly and eliminates the deconfining phase transition for sufficiently light quarks.

\section{Introduction}

We have developed[1] two simple models for the $S U(N)$ gluon plasma equation of state, both based on the use of the Polyakov loop $P$ as the natural order parameter of the deconfinement transition in pure gauge theories[2. In these models, the eigenvalues of the Polyakov loop are the essential degrees of freedom rather than the Polyakov loop trace alone, a possibility also recently explored by Pisarski [3].

Since $P$ is unitary, we can parametrize its diagonal form by $N$ phases $\theta_{j}$ which we refer to as eigenvalues. In our models, confinement is obtained from a set of spatially constant eigenvalues which make $\langle\operatorname{Tr} P\rangle=0$. This is naturally obtained from a uniform distribution of eigenvalues around the unit circle, constrained by the unitary of $P$. In a pure gauge theory below the deconfinement temperature $T_{d}$, the eigenvalues are frozen in this uniform distribution. As $T$ moves upward from $T_{d}$, the eigenvalues of the Polyakov loop rotate towards $\theta=0$ or one of its $Z(N)$ equivalents, and $P$ moves towards an element of the center. In the case of a first order transition, the eigenvalues jump at $T_{d}$. This motion of the eigenvalues

\footnotetext{
*We gratefully acknowledge the support of the U.S. Dept. of Energy under DOE DE-FG02-91ER40628
}

is responsible for the approach to the blackbody limit over the range $T_{d}-5 T_{d}$.

As a consequence of asymptotic freedom, the perturbative expression for the free energy as a function of the Polyakov loop eigenvalues will be valid at sufficiently high temperatures. At one-loop order, the result has the form $f_{\text {pert }}=$ $T^{4} F_{4}\left(\theta_{j}\right)$ where $F_{4}$ has a simple expression in terms of the fourth Bernoulli polynomial; $f_{\text {pert }}$ gives the blackbody behavior expected at high temperature. Note that $f_{\text {pert }}$ is not a function of $\langle\operatorname{Tr} P\rangle$, instead depending directly on the eigenvalues. This distinction begins to matter with $S U(4)$, because for $N \geq 4$, the eigenvalues cannot be determined solely from $\langle\operatorname{Tr} P\rangle$.

\section{Models A and B}

The first of our two models is obtained by adding, by hand, a mass $M$ to the gauge bosons, and working with the high temperature expansion of the resultant free energy to order $M^{2} T^{2}$. The result is

$f_{A}(\theta)=T^{4} F_{4}\left(\theta_{j}\right)-M^{2} T^{2} F_{2}\left(\theta_{j}\right)$

where $F_{2}$ is given as sums of second Bernoulli polynomials [4]. We stress that this derivation merely indicates the type of additional terms that might appear in the free energy. The Bernoulli 
polynomials appear naturally as class functions which are almost everywhere polynomials in the $\theta$ 's, and are well-suited for the construction of a Landau theory in the eigenvalues.

Our second model is obtained by supposing that there is a natural scale $R$ in position space over which color neutrality is enforced. In other words, net color is allowed in volumes of less than $R^{3}$, but the net color on larger scales is zero. This leads to

$f_{B}(\theta)=f_{\text {pert }}(\theta)-\frac{1}{\beta R^{3}} \ln [J(\theta)]$

where $J(\theta)$ is the Jacobian associated with Haar measure on $S U(N)$.

In both models, the $T^{4}$ term, which favors the deconfined phase, dominates for large $T$. The other term, which favors the confined phase, dominates for small $\mathrm{T}$. The deconfinement phase transition results from a conflict between the two terms.

\section{3. $S U(N)$ Thermodynamics for $N=2-5$}

In the case of $S U(2)$ and $S U(3)$, model A is analytically tractable. The deconfining transition is second order for $S U(2)$ and first order for $S U(3)$, in accord with universality arguments [2]. Numerical analysis for the cases of $S U(4)$ and $S U(5)$ show that the deconfinement transition is first order; recent lattice results for $S U(4)$ also indicate a first order transition[5]. Identical results on the order of the transition are obtained numerically for model B.

In the case of $S U(3)$, both models show good agreement with lattice simulations [6] upon comparing the pressure $p$, the energy density $\varepsilon$, and the dimensionless interaction measure $\Delta=(\varepsilon-$ $3 p) / T^{4}$ as a function of the dimensionless variable $T / T_{d}$. The range of temperatures over which metastable behavior occurs can be easily determined, and confirms the weakly first order character of the transition.

It is enlightening to plot $p, \varepsilon$, and $\Delta$, each divided by $N^{2}-1$, versus $T / T_{d}$ for $N=2,3,4$, and 5. Figures 1-3 show the results for model A; model B is similar. Both models appear to quickly approach a large- $N$ limit. Both models show power law behavior in $\Delta$ for sufficiently

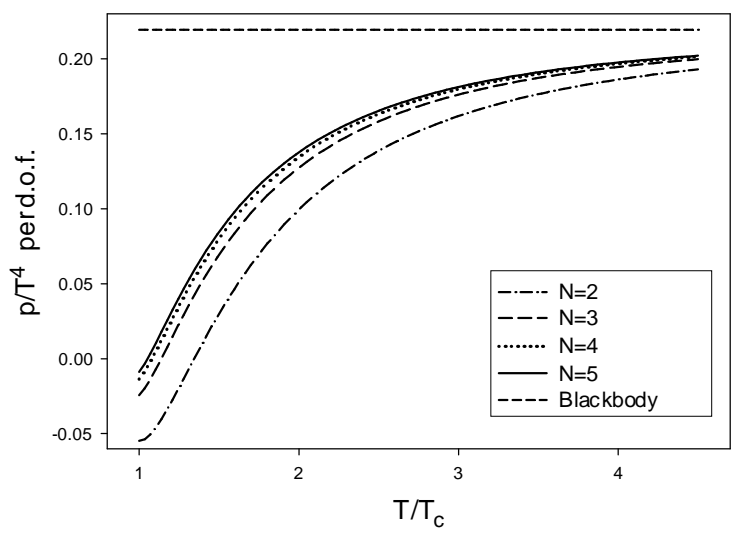

Figure 1. $p / T^{4}$ versus $T / T_{c}$ for model A.

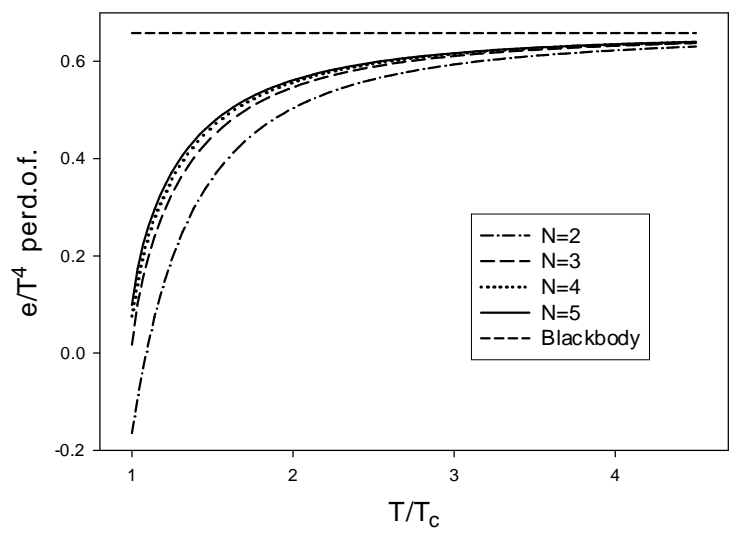

Figure 2. $e / T^{4}$ versus $T / T_{c}$ for model A.

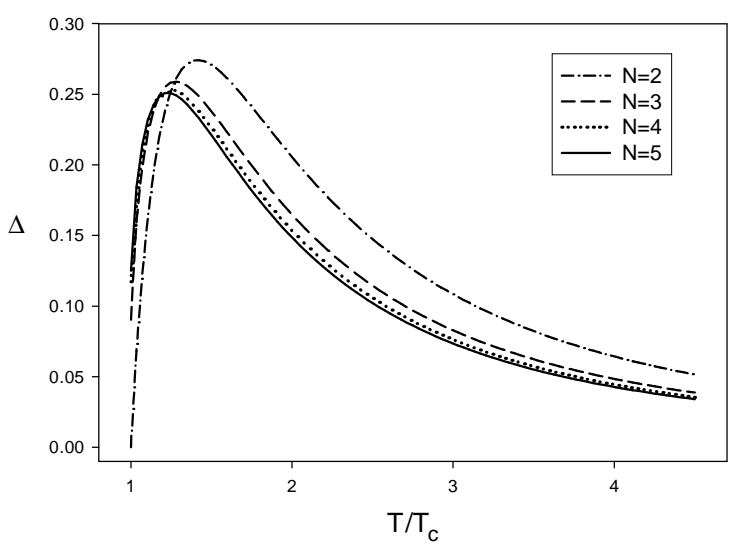

Figure 3. $\Delta$ versus $T / T_{c}$ for model A. 
large $T$, consistent with $\Delta \propto 1 / T^{2}$. This is the asymptotic behavior found analytically for $S U(2)$ in model A, and is compatible with the $S U(3)$ lattice data. In camparison, the Bag model[7], predicts a $1 / T^{4}$ behavior for $\Delta$, which is ruled out by lattice results.

\section{Quarks}

Quark effects can be included as the free energy of quarks propagating in a constant Polyakov loop background. We limit ourselves here to a discussion of very heavy quarks [8] and the leading order effect of light quarks, which do not depend on chiral symmetry effects 911 . The expected effect of very heavy quarks is to lower the critical temperature [12,13]. This line of first-order critical points in the $T-m$ plane terminates in a secondorder end point at some finite value of $m$. For light quarks, we expect that the deconfinement transition is replaced by a smooth crossover with a rapid rise in all thermodynamic quantities. We have confirmed both sets of behaviors in the case of $S U(3)$.

\section{Conclusions}

We have developed two simple phenomenological equations of state for the quark-gluon plasma, which reproduce much of the thermodynamic behavior seen in lattice simulations. Both models correctly predict the order of the deconfining phase transitions for $S U(2)$ (second order) and $S U(3)$ (weakly first order), and predict first order transitions in $S U(4)$ and $S U(5)$ as well. Both models appear to have smooth large- $N$ limits. The numerical value of the parameters introduced are reasonable for both models in the case of $S U(3)$. The deconfinement temperature in a pure $S U(3)$ gauge theory is about $270 \mathrm{MeV}$, giving a value for $M$ of $596 \mathrm{MeV}$, a plausible value for a constituent gluon mass. In model B, we find that $R$ is 1 fermi. It is clear that by allowing the parameters $M$ and $R$ to depend on the temperature, a better fit to lattice data can be obtained at the cost of introducing additional phenomenological parameters.

The success of these phenomenological mod- els suggests a point of view on the nature of the deconfinement mechanism. In confining theories, the eigenvalue distributions of the Polyakov loop are peaked at low temperature around values evenly spaced about the unit circle in such a way that the expectation value of the Polyakov loop is zero. At the deconfining transition temperature, the peaks of the eigenvalue distributions move towards 1 , which is the asymptotic limit as $T$ goes to infinity. We believe that this picture of deconfinement coupled with a field-theory inspired model of chiral symmetry breaking has the potential to fully model the equation of state of the quark-gluon plasma 14.15 .

\section{REFERENCES}

1. P. N. Meisinger, T. R. Miller and M. C. Ogilvie, hep-ph/0108009.

2. L. G. Yaffe and B. Svetitsky, Phys. Rev. D 26, 963 (1982).

3. R. D. Pisarski, Phys. Rev. D 62, 111501 (2000) hep-ph/0006205.

4. P. N. Meisinger and M. C. Ogilvie, hep$\mathrm{ph} / 0108026$.

5. M. Wingate and S. Ohta, Phys. Rev. D 63, 094502 (2001) hep-lat/0006016.

6. G. Boyd, J. Engels, F. Karsch, E. Laermann, C. Legeland, M. Lutgemeier and B. Petersson, Nucl. Phys. B 469, 419 (1996) heplat/9602007.

7. J. Cleymans, R. V. Gavai and E. Suhonen, Phys. Rept. 130, 217 (1986).

8. P. N. Meisinger and M. C. Ogilvie, Phys. Rev. D 52, 3024 (1995) hep-lat/9502003.

9. D. J. Gross, R. D. Pisarski and L. G. Yaffe, Rev. Mod. Phys. 53, 43 (1981).

10. N. Weiss, Phys. Rev. D 24, 475 (1981).

11. N. Weiss, Phys. Rev. D 25, 2667 (1982).

12. F. Green and F. Karsch, Nucl. Phys. B 238, 297 (1984).

13. M. Ogilvie, Phys. Rev. Lett. 52, 1369 (1984).

14. P. N. Meisinger and M. C. Ogilvie, Nucl. Phys. Proc. Suppl. 47, 519 (1996) heplat/9509050].

15. A. Dumitru and R. D. Pisarski, Phys. Lett. B 504, 282 (2001) hep-ph/0010083. 